\title{
Meta-analysis of the Value of Cardiac Nuclear Magnetic Resonance in the Diagnosis of Viral Myocarditis
}

\author{
Juan Xu and Yikai Xu \\ Department of Medical Imaging Centre, Nanfang Hospital, Southern Medical University, 1838 Guangzhou, China
}

\begin{abstract}
To evaluate the diagnostic value of cardiac magnetic resonance imaging (MRI) in viral myocarditis by meta-analysis. PubMed, EMbase, Cochrane, Web of Science, China National Knowledge Infrastructure (CNKI, China) Wanfang Databases were searched for clinical research literature on MRI diagnosis of viral myocarditis from the establishment of the database to March 1 , 2020.The pooled sensitivity, specificity, positive likelihood ratio, negative likelihood ratio, diagnostic odds ratio (DOR) and the summary receiver operating characteristic (SROC) were analysed by Stata 15.0. Eight articles were included in the end. The results showed that the sensitivity, specificity, DOR, and area under the curve (AUC) SROCs were 94\%, 75\%, 45.24 and 0.88, respectively. Existing research have confirmed that cardiac MRI has high sensitivity (94\%) and moderate specificity (75\%) for viral myocarditis. The positive ratio of test in myocarditis group is 45.24 times higher than that in non-myocarditis group indicating the outstanding effect of diagnosis and discrimination.
\end{abstract}

Key Words: Cardiac magnetic resonance imaging, Viral myocarditis, Systematic review, Meta-analysis.

How to cite this article: Xu J, Xu Y. Meta-analysis of the Value of Cardiac Nuclear Magnetic Resonance in the Diagnosis of Viral Myocarditis. J Coll Physicians Surg Pak 2020; 30(12):1326-1331.

\section{INTRODUCTION}

The occurrence of myocarditis is common in adolescents. The American Epidemiological Survey found that the incidence of myocarditis was 22 in 100,000, and the mortality rate was 4.8 in $100,000 .^{1,2}$ There are a great number of influence factors of myocarditis, such as age, gender, region, race, virus strain and genetic susceptibility of population. During the 3-year followup, it was found that $21 \%$ of the patients with myocarditis had delayed course of disease, resulting in chronic myocarditis and dilated cardiomyopathy (DCM). ${ }^{1,3}$ The common pathogenic factors of myocarditis are viral infection, including Enterovirus, Adenovirus, Parvovirus B19 (PVB 19), Herpes virus 6 (HPV6) and Cytomegalovirus. The most common type was Coxsackie virus group B, type 3 (Coxsackievirus B3, CVB3). ${ }^{4}$

The pathophysiological changes of viral myocarditis (VMC) can be divided into three stages. The first stage is acute phase, in which after the binding of virus and the viral receptor on the cardiomyocytes surface, endocytosis enters into cardiomyocytes, directly causing the toxic reaction of cardiomyocytes and pyrolysis necrosis.

Correspondence to: Dr. Yikai Xu, Department of Medical Imaging Centre, Nanfang Hospital, Southern Medical University, 1838 Guangzhou Ave.N., 510515, P.R. China E-mail: ca65469@163.com

Received: March 16, 2020; Revised: May 26, 2020; Accepted: June 13, 2020

DOI: https://doi.org/10.29271/jcpsp.2020.12.1326
The second stage is subacute phase, which, following the previous stage, is the occurrence of the activation of autoimmune response (natural immunity, humoral immunity and cellular immunity), activation of inflammatory cells such as macrophages and Tcells, release of a large number of inflammatory factors and aggravation of cardiomyocyte apoptosis and necrosis. Inflammatory cells release a variety of matrix hydrolases, myocardial interstitial extracellular matrix degradation, resulting in cardiac function damage and ventricular dilatation. The third stage is chronic phase, the virus continues to replicate with low titer and progresses to chronic dilated cardiomyopathy.

Most patients with viral myocarditis have mild clinical symptoms, but a few may have explosive myocarditis or delayed dilated cardiomyopathy. ${ }^{5,6}$ It was found that patients with severe myocarditis were complicated with significant hemodynamic abnormalities, with pathology showing numerous inflammatory cell infiltration, and the long-term favorable prognosis after recovery in a short time. However, the virus persisted with less inflammatory infiltration and progressed to chronic viral myocarditis or dilated cardiomyopathy with poor prognosis. ${ }^{7,8} \mathrm{The}$ diagnostic methods of viral myocarditis include clinical manifestations, serological markers, electrocardiogram, cardiac ultrasound, MRI and endomyocardial biopsy. At present, there is no single clinical or imaging method to confirm the diagnosis of viral myocarditis. Biopsy is still the golden standard for the diagnosis of the disease, but being at greatriskin practical application, myocardial biopsy is a moderately traumatic inspection, and has blind areas of observation, especially in children, and limited sensitivity to sampling of focal myocarditis. According to the literature, the sensitivity is only about $25 \%,{ }^{9}$ followed by thin myocardium, which 
increases the incidence of complications such as perforation and pericardial tamponade. Currently, the MRI uses Lake Louise Consensus Criteria as main diagnostic criteria of myocarditis. Whereas, MRI is a non-invasive examination method, which has the characteristics of multi-parameter and multi-directional imaging, good soft tissue contrast and high spatial resolution. It can not only show the location of myocardial injury, but also the degree ofmyocardialedema, which has great potential in the diagnosis of myocarditis. ${ }^{10,11}$ In this study, a systematic evaluation method was used to evaluae the value of cardiac MRI in the diagnosis of VMC, so as to provide a basis for the diagnosis of VMC.

\section{METHODOLOGY}

Research was selected, based on the accuracy of MRI in the diagnosis of VMC, with four tables of data available for extraction, languages limited to English and Chinese. Research subjects should have been patients suspected or diagnosed with acute or chronic VMC or myocarditis, having typical history of prodromal infection, corresponding clinical manifestations, electrocardiogram, markers of myocardial injury, and evidence of myocardial injury shown by echocardiography, or endomyocardial biopsy. The diagnostic method to be evaluated was MRI diagnosis, and the gold standard was subendocardial myocardial biopsy. Cardiac MRI diagnosis of viral myocarditis according to Lake Louis standard. ${ }^{12}$

Measurement indicators were combined sensitivity (SEN combined), combined specificity (SPEcombined), combined positive likelihood ratio (+ LR combined), combined negative likelihood ratio (-LR combined), combined diagnostic odds ratio (DOR combined), area under the SROC curve (AUC). Excluding standard was studies using animal experiments, and studies with incomplete reported diagnostic accuracy data.

MRI diagnosis of viral myocarditis clinical research literature were searched from PubMed, EMbase, Cochrane, Web of science, China National Knowledge Infrastructure (CNKI), China Wanfang database from the establishment date to March 1,2020. The search terms were (Cardiac Magnetic Resonance OR CMR) AND (viral myocarditis OR VMC OR myocarditis) AND diagnosis. The research will trace the relevant literature in clinical trials or review references, and contact the author as far as possible to askfor the relevant literature.

Two evaluators independently screened the literature, extracted the data and cross-checked according to the pre-established inclusion and exclusion criteria. Inconsistencies were decided through discussion. The two researchers designed the tables and extracted data from several aspects including included subjects (gender, age, disease, diagnostic criteria, inclusion criteria, exclusion criteria, etc. ), tests remained to be evaluated (instruments, test methods, blinding methods, detailed operational process reports), control trials (reference standard positive standards, detection methods, blind methods, etc.), results (sensitivity, specificity, number of true positive, false positive, false negative, and true negative).
The methodological quality evaluation of clinical trials was carried out independently by the two researchers according to the quality assessment of diagnostic accuracy studies(QUADAS) standards developed by Whiting. ${ }^{13}$ When there was disagreement, the quality of the literature was solved by discussion. Each criterion was evaluated by "Yes" (meet the criteria), "No" (not satisfy the criteria or not mentioned) and "Unclear" (partially satisfy or cannot obtain sufficient information from the literature). The QUADAS scale evaluates each article from three aspects: bias $(3 \sim 7,10 \sim 12,14)$, variation $(1,2)$ and report quality $(8,9,13)$, and figures out the causes of all kinds of bias and variation.

Stata 15.0 was used to analyse the data. Heterogeneity analysis was first conducted, using $\mathrm{I}^{2}$ to evaluate heterogeneity. If $\mathrm{I}^{2}$ $<50 \%$, it is considered that the heterogeneity between the research results is low. If $\mathrm{I}^{2} \geq 50 \%$, there is a high degree of heterogeneity between the research results. If the same heterogeneity between different diagnostic tests of a diagnostic method was relatively small, the Stata was used to draw the SROC curve, and the combined sensitivity, specificity, positive likelihood ratio, negative likelihood ratio and DOR were calculated respectively. If there is heterogeneity, the first step is to analyse the source of heterogeneity. If the heterogeneity is resulted from large methodological quality difference of different research, the sensitivity analysis will be carried out.

\section{RESULTS}

Four hundred and ninety-two studied were collected through tentative search. After reading the title and abstract, 418 irrelevant literature were excluded, with further exclusion of 66 literature with incomplete information via reading the full text. Finally, 8 literature were included. ${ }^{14-21}$ All of them were clinical controlled trials, with a total of 448 patients. The flow chart of document retrieval (Figure 1). The basic characteristics and quality evaluation of literature inclusion were shown in Table I. Thus it could be seen that all the QUADAS scores included in the study were greater than or equal to 9 .

The ROC plane scatter plot output by Stata 15.0 software showed atypical shoulder arm shape-(Figure 2). Spearman test showed $p=0.1942$, indicating that there was no heterogeneity caused by threshold effect. The results of heterogeneous sources showed that there was heterogeneity in sensitivity ( $p$ $\left.<0.001, I^{2}=87.29 \%\right)$, specificity $\left(p=0.01, I^{2}=63.09 \%\right)$, positive likelihood ratio $\left(p=0.02, I^{2}=25.96 \%\right)$, negative likelihood ratio $\left(P=0.00, I^{2}=81.42 \%\right)$, The DOR ( $\left.p<0.001, I^{2}=89.60 \%\right)$; thus, the random effect model was used for meta-analysis.

The results showed that combined sensitivity, specificity, + LR, -LR, and DOR and SROC AUC were 0.94 (95\% Cl: 0.78 0.98), 0.75 (95\% Cl: $0.63 \sim 0.85)$, $3.82(95 \% \mathrm{Cl}: 2.58 \sim 5.65), 0.08(95 \%$ Cl: $0.02 \sim 0.30), 45.24(95 \% \mathrm{Cl}: 13.38 \sim 152.96), 0.88(95 \% \mathrm{Cl}:$ $0.85 \sim 0.90)$, respectively. The forest maps of DOR, sensitivity and specificity, positive likelihood ratio and negative likelihood ratio were shown in Figures 3-5. 
Table I: Characters of included studies.

\begin{tabular}{|c|c|c|c|c|c|c|c|c|c|c|}
\hline First author & Country & Year & MRI scanner brand & Cases of viral myocarditis & Similar cases & Tp & Fp & Fn & Tn & QUADAS score \\
\hline Friedrich & Germany & 1998 & GE Healthcare & 16 & 21 & 16 & 3 & 0 & 18 & 9 \\
\hline Laissy & France & 2002 & Siemens & 10 & 17 & 10 & 10 & 0 & 7 & 10 \\
\hline Hyafil & France & 2005 & GE Healthcare & 27 & 20 & 21 & 5 & 6 & 15 & 9 \\
\hline Mathrholdt & Germany & 2006 & Siemens & 89 & 39 & 89 & 13 & 0 & 26 & 11 \\
\hline Matthias & Germany & 2008 & GE Healthcare & 40 & 43 & 35 & 13 & 5 & 30 & 8 \\
\hline OuYang HC & China & 2014 & philips & 19 & 11 & 16 & 2 & 3 & 9 & 9 \\
\hline Ran H & China & 2017 & Siemens & 16 & 5 & 15 & 1 & 1 & 4 & 9 \\
\hline Sebai & French & 2019 & Philips & 44 & 31 & 25 & 2 & 19 & 29 & 11 \\
\hline
\end{tabular}

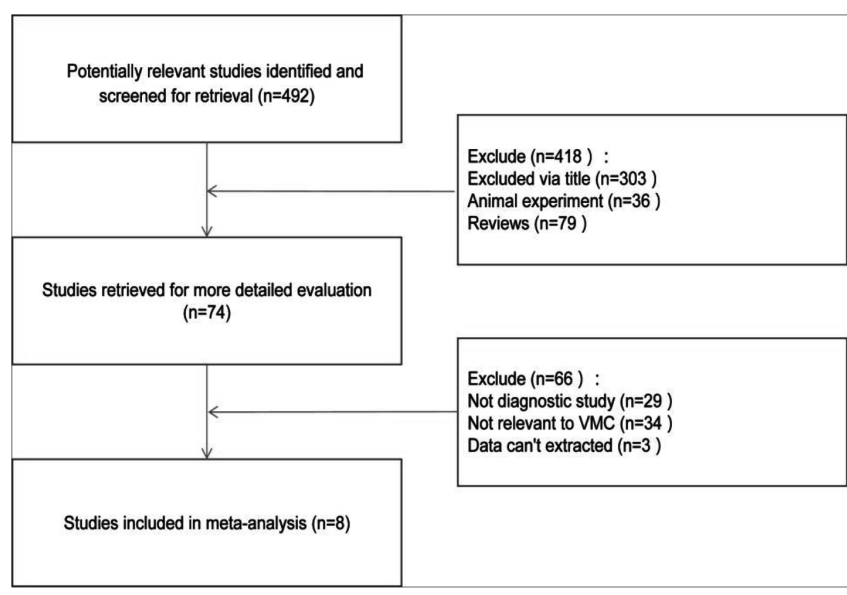

Figure1: A flow diagram of the study selection process.

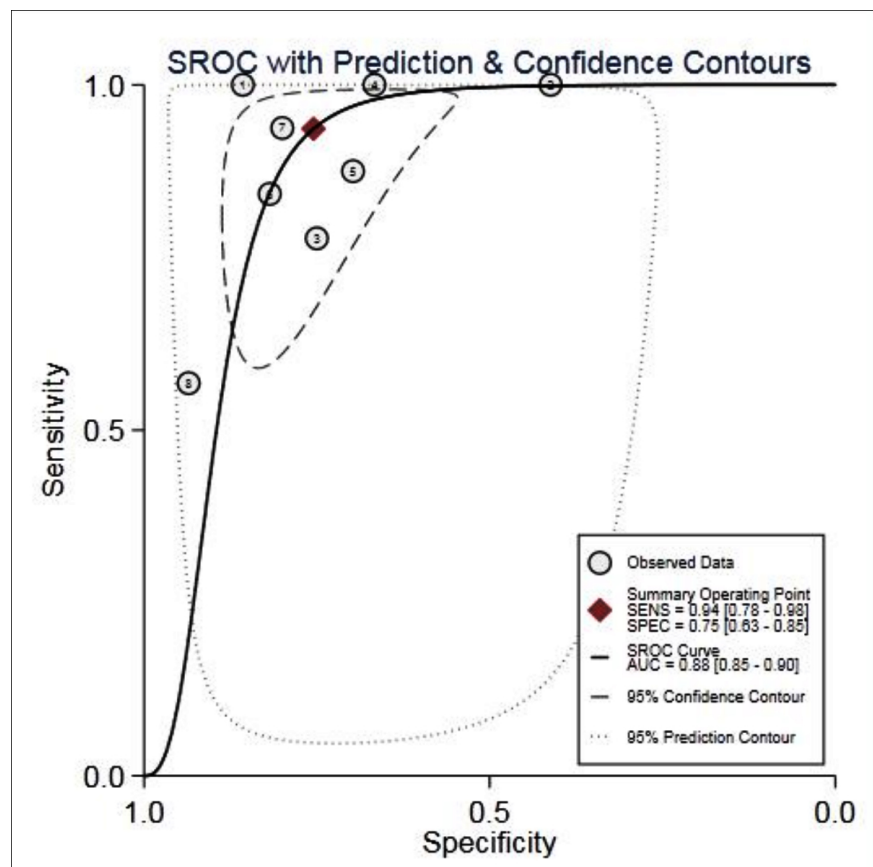

Figure 2: SROC curve for the accuracy of MRI in the diagnosis of VMC.

The inclusion study did not show significant publication bias (Figure 6). The subgroup analysis of national and MRI scanners showed that the heterogeneity did not decrease significantly. The sensitivity analysis was carried out after each study was excluded one by one. The results showed that there was no significant change in the combined DOR, implying that the stability of the literature included was high.

The results of the publication bias (Figure 6 ) indicated that the difference had no statistically significant $(p=0.45)$, which could be considered as no publication bias in the included study.

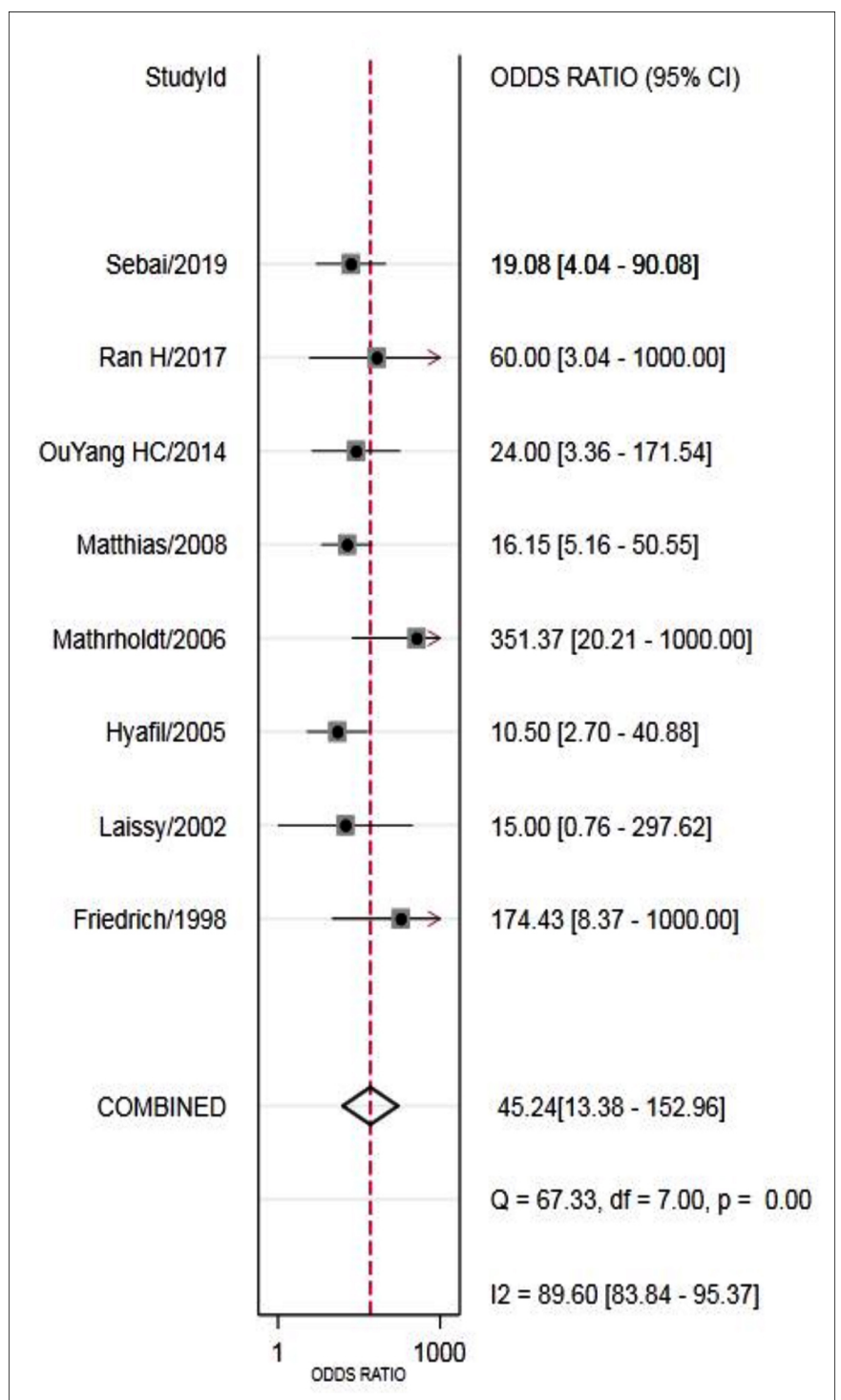

Figure 3: Forest plot of DOR of MRI for the diagnosis of VMC. 


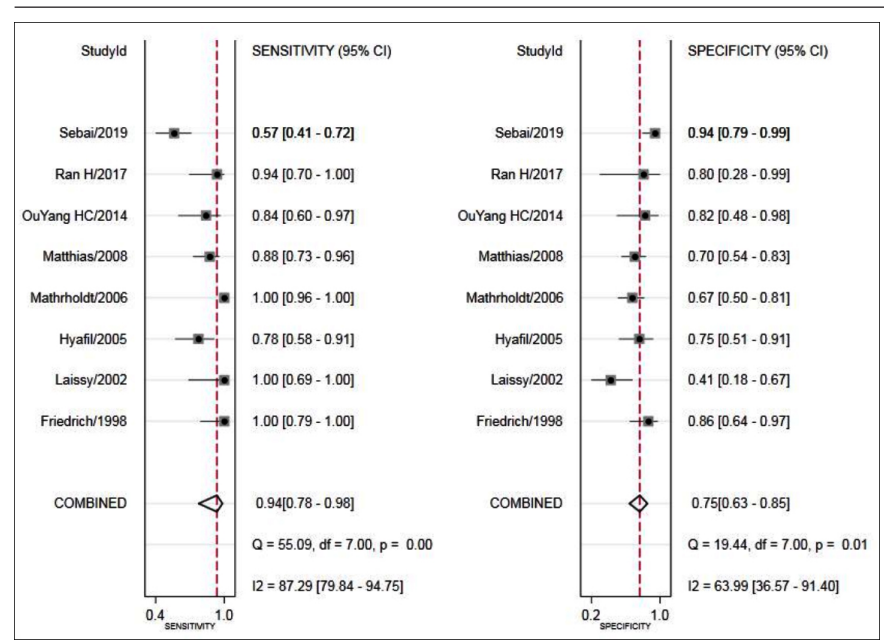

Figure 4: Forest plot of sensitivity and specificity of MRI for the diagnosis of VMC.

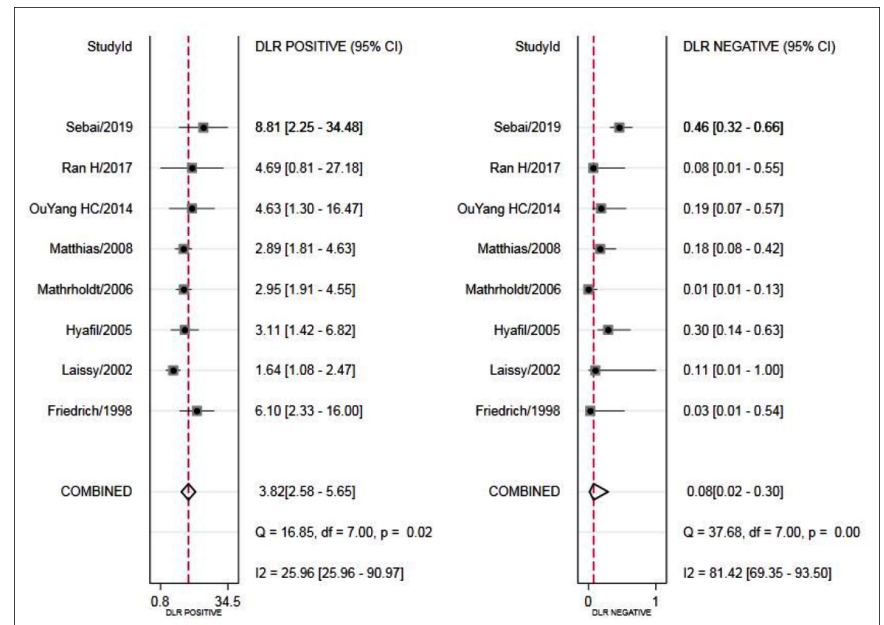

Figure 5: Forest plot of LR+ and LR- of MRI for the diagnosis of VMC.

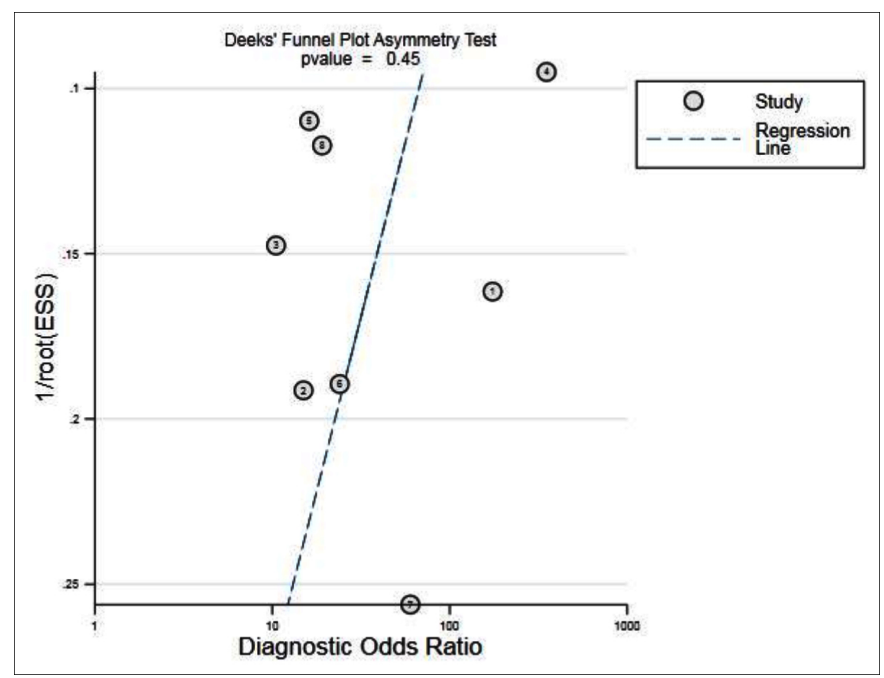

Figure 6: Results of publication bias.

\section{DISCUSSION}

The golden standard for the diagnosis of viral myocarditis is myocardial biopsy, but this is an invasive examination, which is not conducive to the early diagnosis of patients. In case of ensuring a certain degree of specificity, there can be a certain degree of sensitivity of cardiac MRI examination, early detection of viral myocarditis, it is particularly important, because it is non-invasive. This has very important clinical significance. Viral myocarditis patients are mainly caused by the virus invasion of cardiomyocytes. Most of the patients have upper respiratory tract or thoracic viral infection before the onset of illness. This type of disease can easily lead to myocardial dysfunction and induce heart failure or sudden death, which has a great effect to the health of patients. ${ }^{22}$ In the process of clinical diagnosis, due to its multiple clinical symptoms and the unobvious specificity, it is easy to be confused with other noninfectious myocardial diseases, leading to misdiagnosis.

The cardiac intimal biopsy can be used to accurately determine whether the myocardium of the patient is infected with virus, the type of virus infection; thus, the technique has also become the gold standard for the diagnosis of viral myocarditis. However, due to the necessity of invading into the heart during the process of diagnosis, the technology will inevitably cause damage to the myocardium and surrounding tissues, which will also have certain impact on the recovery of the patient from disease. Therefore, it is usually used in severe patients with no remission after routine treatment. MRI has the advantages of multi-direction, multi-parameter and accurate imaging, which is also the most accurate non-invasive myocarditis diagnostic technique currently. According to the statistics from the World Health Organization (WHO), over $30 \%$ of suspected viral myocarditis patients in Europe tend to choose MRI for diagnosis.

Pathological studies have shown that during the early stage of onset of patient (about 3 weeks), the edema of cardiomyocyte will reach its maximum; meanwhile, the T2-weighted signal will also increase significantly under MRI. Therefore, acute and chronic myocarditis can be distinguished through the inspection of this signal, which can be applied to effectively assess the situation of ischemic injury of patients with viral myocarditis. However, since T2-weighted imaging can be greatly affected by external low signal noise, it cannot serve as a single index to estimate the condition of patient, requiring the combination with other diagnostic data. ${ }^{22}$ Simultaneously, when the patient has acute viral myocarditis, there will be obvious myocardial injury, accompanied by cytolysis/cell dissolution, vasodilation, inflammatory intralesional hypervolemia, etc., resulting in an increase in the proportion of contrast media absorbed by blood vessels. Thus, alternative method can be delaying imaging to judge whether there is myocardial injury by comparing the beforeand-after images. However, this type of examination can be easily affected by arrhythmia and respiratory incoordination, and the probability of artifact in the image is relatively high, so it cannot be a single judgment index as well. ${ }^{23}$ The evaluation of the clinical value of diagnostic trials is mainly 
conducted from several aspects, including object of the study, reference standards, the trials to be evaluated and the controlled trials, the report quality of hinding and experiment of the control trial results, in order to explore the scale and source of bias and heterogeneity, to guide the clinical selection of favourable/great diagnostic tests, and to enable make an appropriate interpretation of the test results.

In this systematic review, 448 patients were included. The results of statistical analysis showed that the combined sensitivity and specificity of VMC diagnosed by cardiac MRI were $94 \%$ and $75 \%$, respectively, indicating that the omission diagnosis rate was $6 \%$ and the misdiagnosis rate was $25 \%$. The combined + LR was 3.82>1, suggesting that when the result of cardiac MRI was positive, the possibility of developing VMC was higher, and the combined -LR was $0.08<0.1$, signifying that when the result of cardiac MRI was negative, the possibility of excluding VMC was higher. The AUC of the SROC was 0.88 , indicating that the diagnostic efficiency was relatively high.

Certainly, there were some limitations in the study. For instance, there was heterogeneity in the study. Considering the possible differences in the design between the case group and the control group, part of the literature, ${ }^{19,20}$ only have healthy population in the control group. In addition, differences in ethnicity, medical background, disease level, and whether received treatment can also produce heterogeneity. Changes in each composition of detecting instruments, operation procedures, quality control, and operator operating instruction may impact the accuracy of laboratory results.

\section{CONCLUSION}

The evaluation of this system suggested that cardiac MRI had a relatively high sensitivity $(94 \%)$ in the diagnosis of VMC, but the specificity was in the middle level $(75 \%)$. The positive predictive value showed that the probability of the disease was high, and the omission diagnosis rate of suspected cases was only $6 \%$, which was helpful for the screening of the disease. Due to its $25 \%$ misdiagnosis rate, which could not be applied as a diagnosis of disease, and the negative test results were more likely to exclude the disease. The area under the SROC curve was 0.88 , indicating that cardiac nuclear magnetic resonance as a non-invasive examination is sensitive to the diagnosis of myocarditis and can be used as a screening method. Considering that there are still some limitations in this study, further high-quality research are required to verify the study.

\section{CONFLICT OF INTEREST:}

The authors declared no conflict of interest.

\section{AUTHORS' CONTRIBUTION:}

JX, YX: Contributions to the conception or design of the work, drafting the work or revising it critically for important intellectual content, final approval of the version to be published.

\section{REFERENCES}

1. Heymans S, Eriksson U, Lehtonen J, Cooper LJ. The quest for new approaches in myocarditis and inflammatory cardiomyopathy. J Am Coll Cardiol 2016; 68(21):2348-64. doi: 10.1016/j.jacc.2016.09.937.

2. Subahi A, Akintoye E, Yassin AS, Abubakar H, Adegbala O, Mishra $T$, et al. Impact of atrial fibrillation on patients hospitalised for acute myocarditis: Insights from a nationally-representative United States cohort. Clin Cardiol 2019; 42(1):26-31. doi: 10.1002/clc.23088.

3. Besler C, Urban D, Watzka S, Lang D, Rommel KP, Kandolf R, et al. Endomyocardial miR-133a levels correlate with myocardial inflammation, improved left ventricular function, and clinical outcome in patients with inflammatory cardiomyopathy. EurJ Heart Fail 2016; 18(12): 1442-51. doi: 10.1002/ejhf.579.

4. Tschope C, Muller I, Xia Y, Savvatis K, Pappritz K, Pinkert S, et al. NOD2 (Nucleotide-binding oligomerisation domain 2) is a major pathogenic mediator of coxsackievirus b3-induced myocarditis. Circ Heart Fail 2017; 10.

5. Luo $\mathrm{H}$, Wong J, Wong B. Protein degradation systems in viral myocarditis leading to dilated cardiomyopathy. Cardiovasc Res 2010; 85(2):347-56. doi: 10.1093/cvr/ cvp225.

6. Goldberg L, Tirosh-Wagner T, Vardi A, Abbas H, Pillar N, Shomron N, et al. Circulating MicroRNAs: A potential biomarker for cardiac damage, inflammatory response, and left ventricular function recovery in pediatric viral myocarditis. J Cardiovasc Transl Res 2018; 11(4):319-28. doi: 10.1007/s12265-018-9814-0.

7. Klingel K, Kandolf R. Osteopontin: A biomarker to predict the outcome of inflammatory heart disease. Semin Thromb Hemost 2010; 36(2):195-202. doi: 10.1055/s-0030- 1251504.

8. Andreoletti L, Leveque N, Boulagnon C, Brasselet C, Fornes P. Viral causes of human myocarditis. Arch Cardiovasc Dis 2009; 102(6-7) 559-68. doi: 10.1016/j.acvd.2009.04.010.

9. Mavrogeni S, Bratis K, Georgakopoulos D, Karanasios E, Kolovou G, Pavlides G, et al. Evaluation of myocarditis in a pediatric population using cardiovascular magnetic resonance and endomyocardial biopsy. Int J Cardiol 2012; 160(3):192-95. doi: 10.1016/j.ijcard.2011.04.019.

10. Narducci ML, Pelargonio G, La Rosa G, Inzani F, D'Amati G, Novelli $V$, et al. Role of extensive diagnostic workup in young athletes and nonathletes with complex ventricular arrhythmias. Heart Rhythm 2020; 17(2):230-37. doi: 10.1016/j.hrthm.2019.08.022.

11. Baessler B, Luecke C, Lurz J, Klingel K, Das A, von Roeder M, et al. Cardiac MRI and texture analysis of myocardial T1 and T2 maps in myocarditis with acute versus chronic symptoms of heart failure. Radiology 2019; 292(3):608-17. doi: 10.1148/radiol.2019190101.

12. Friedrich MG, Sechtem U, Schulz-Menger J, Holmvang G, Alakija P, Cooper LT, et al. Cardiovascular magnetic resonance in myocarditis: A JACC White Paper. J Am Coll Cardiol 2009; 53:1475-87.

13. Whiting PF, Weswood ME, Rutjes AW, Reitsma JB, Bossuyt PN, Kleijnen J. Evaluation of QUADAS, a tool for the quality assessment of diagnostic accuracy studies. BMC Med Res 
Methodol 2006; 6:9. doi: 10.1186/1471-2288-6-9.

14. Gutberlet M, Spors B, Thoma T, Bertram H, Denecke T, Felix $\mathrm{R}$, et al. Suspected chronic myocarditis at cardiac MR: Diagnostic accuracy and association with immuno-histologically detected inflammation and viral persistence. Radiology 2008; 246(2):401-09. doi: 10.1148/radiol. 2461062179.

15. Haichun $\mathrm{O}$, Haixiong $C$, Yunzhao $H$, Yanxian W, Wensheng $L$, Yuying $C$, et al. The diagnostic value of MRI in acute viral myocarditis. Chinese J Cardiol 2014; 42:927-31.

16. Hua R, Zhengzhong W, Yi H, Wanchun G, Aijun S, Jiaquan Z. Cardiac magnetic resonance imaging as a diagnostic tool in viral myocarditis with the main symptoms of acute coronary syndrome. Chinese J Magnetic Resonance Imaging 2017; 8:578-82.

17. Sebai F, Brun S, Petermann A, Ribes D, Prevot G, Cariou E, et al. Cardiac magnetic resonance imaging with late gadolinium enhancement in acute myocarditis: Towards differentiation between immune-mediated and viral-related aetiologies. Arch Cardiovasc Dis 2019; 112(10):559-66. doi: 10.1016/j.acvd.2019.09.001.

18. Friedrich MG, Strohm O, Schulz-Menger J, Marciniak H, Luft FC, Dietz R. Contrast media-enhanced magnetic resonance imaging visualizes myocardial changes in the course of viral myocarditis. Circulation 1998; 97(18):1802-09. doi: 10.1161/01.cir.97.18.1802.

19. Laissy JP, Messin B, Varenne O, lung B, Karila-Cohen D, Schouman-Claeys $\mathrm{E}$, et al. MRI of acute myocarditis: A comprehensive approach based on various imaging sequences. Chest 2002; 122(5):1638-48. doi: 10.1378/chest. 122.5.1638.

20. Laissy JP, Hyafil F, Feldman LJ, Juliard JM, Schouman-Claeys E, Steg PG, et al. Differentiating acute myocardial infarction from myocarditis: diagnostic value of early- and delayed-perfusion cardiac MR imaging. Radiology 2005; 237(1): 75-82. doi: 10.1148/radiol.2371041322.

21. Mahrholdt H, Wagner A, Deluigi CC, Kispert E, Hager S, Meinhardt G, et al. Presentation, patterns of myocardial damage, and clinical course of viral myocarditis. Circulation 2006; 114(15):1581-90. doi: 10.1161/CIRCULATIONAHA.105. 606509 .

22. Gannon MP, Schaub E, Grines CL, Saba SG. State of the art: Evaluation and prognostication of myocarditis using cardiac MRI. J Magn Reson Imaging 2019; 49(7):e122-31. doi: 10.1002/jmri.26611.

23. Won E, Donnino R, Srichai MB, Sedlis SP, Feit F, Rolnitzky L, et al. Diagnostic accuracy of cardiac magnetic resonance imaging in the evaluation of newly diagnosed heart failure with reduced left ventricular ejection fraction. Am J Cardiol 2015; 116(7): 1082-87. doi: 10.1016/j.amjcard.2015.06.032. 